\title{
Supra-patellar swelling and knee instability
}

\author{
M. Ehlinger • P. Adam • G. Bierry • J. C. Dosch • \\ G. Taglang $\cdot$ F. Bonnomet
}

Received: 28 October 2009 /Revised: 4 January 2010 /Accepted: 7 January 2010 / Published online: 9 March 2010

(C) ISS 2010

Keywords Knee $\cdot$ Intra-articular tumor $\cdot$ Femoro-patellar instability

\section{Question}

A 22-year-old woman had been suffering from suprapatellar swelling and patellar instability for 1 year.

The diagnosis can be found at doi: 10.1007/s00256-010-0881-9.

M. Ehlinger $(\bowtie) \cdot$ P. Adam $\cdot$ G. Taglang $\cdot$ F. Bonnomet

Service de Chirurgie Orthopédique et de Traumatologie, CHU

Hautepierre, Hôpitaux Universitaires de Strasbourg,

1 Avenue Molière,

67098 Strasbourg Cedex, France

e-mail: matthieu.ehlinger@chru-strasbourg.fr

P. Adam

e-mail: philippe.adam@chru-strasbourg.fr

G. Taglang

e-mail: gilbert.taglang@chru-strasbourg.fr

F. Bonnomet

e-mail: francois.bonnomet@chru-starsbourg.fr

G. Bierry $\cdot$ J. C. Dosch

Service de Radiologie, CHU Hautepierre, Hôpitaux Universitaires de Strasbourg,

1 Avenue Molière,

Strasbourg Cedex, France

G. Bierry

e-mail: guillaume.bierry@chru-strasbourg.fr

J. C. Dosch

e-mail: jeanclaude.dosch@chru-strasbourg.fr

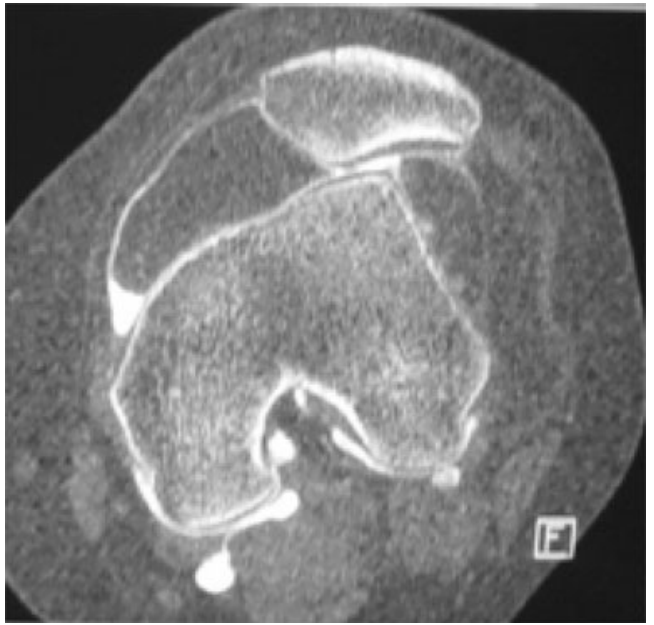

Fig. 1 Arthro-CT scan, axial view

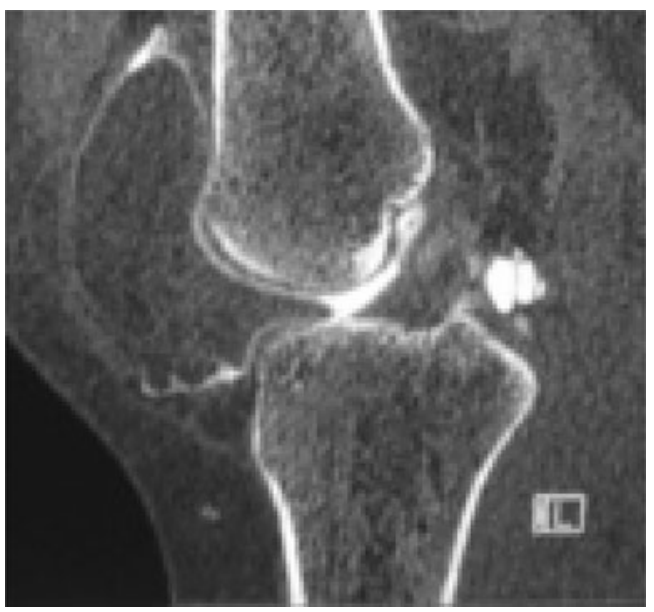

Fig. 2 Arthro-CT scan, sagittal view 


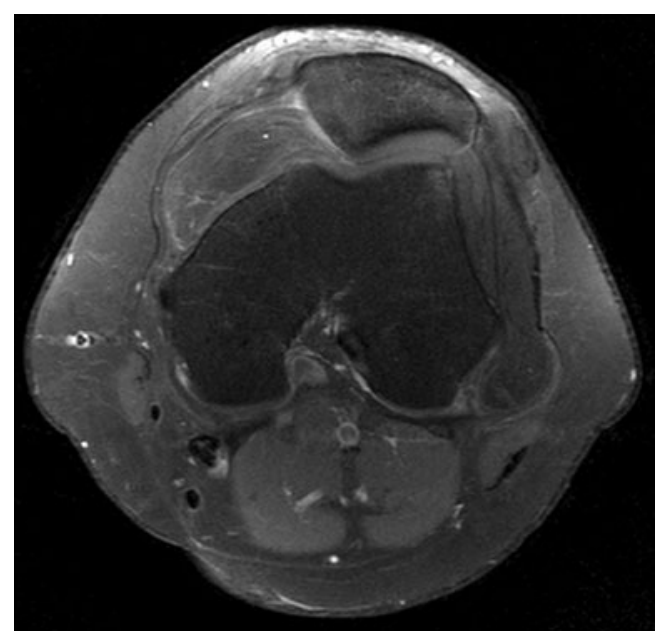

Fig. 3 MRI, axial view, T2-weighted, fat-saturated

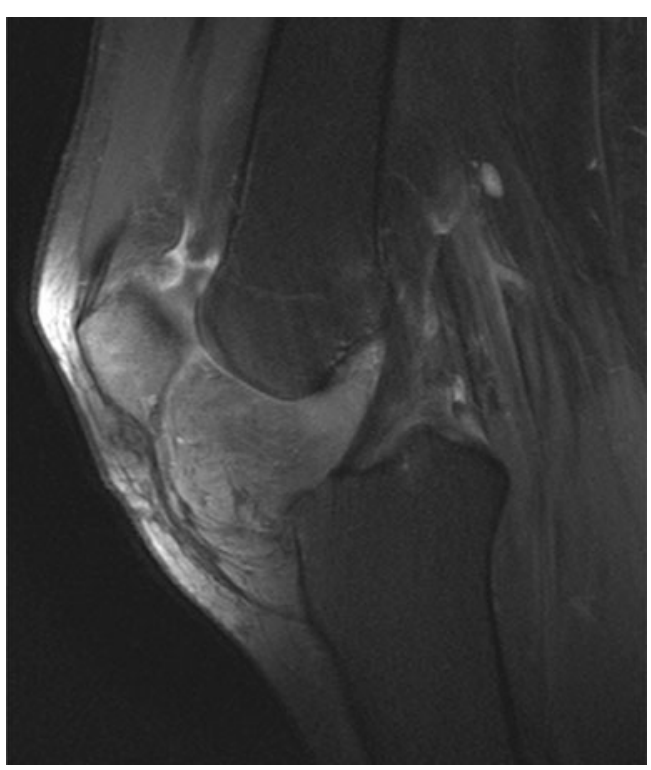

Fig. 4 MRI, median sagittal view, T1-weighted, fat-saturated, after gadolinium administration 